\title{
Phosphoglycerate dehydrogenase is a novel predictor for poor prognosis in gastric cancer
}

This article was published in the following Dove Press journal:

OncoTargets and Therapy

7 September 2016

Number of times this article has been viewed

\author{
Yun Xian ${ }^{1, *}$ \\ Shu Zhang',* \\ Xudong Wang ${ }^{3}$ \\ Jin Qin ${ }^{2}$ \\ Wei Wang ${ }^{2}$ \\ $\mathrm{Han} \mathrm{Wu}^{4}$ \\ 'School of Public Health, Nantong \\ University, ${ }^{2}$ Department of Pathology, \\ ${ }^{3}$ Department of Laboratory Medicine, \\ ${ }^{4}$ Department of General Surgery, \\ Affiliated Hospital of Nantong \\ University, Nantong, Jiangsu, People's \\ Republic of China \\ *These authors contributed equally \\ to this work
}

Purpose: Phosphoglycerate dehydrogenase (PHGDH) acts as a key metabolic enzyme in the rate-limiting step in serine biosynthesis and plays an important role in metastasis of several cancers. The aim of this study was to investigate the prognostic value of PHGDH in gastric cancer (GC).

Methods: The messenger RNA expression of PHGDH was determined in 20 pairs of cancerous and adjacent nontumor tissues by real-time polymerase chain reaction. Immunohistochemistry of PHGDH was performed on tissue microarray, composed of $482 \mathrm{GC}$ and 64 matched adjacent nontumor tissues acquired from surgery, 20 chronic gastritis, 18 intestinal metaplasia, and 31 low-grade and 66 high-grade intraepithelial neoplasias acquired through gastric endoscopic biopsy. Univariate and multivariate Cox proportional hazard models were used to perform survival analyses.

Results: Both PHGDH messenger RNA and protein product exhibited GC tissue-preferred expression, when compared with benign tissues. The high PHGDH expression was significantly correlated with histological type $(P=0.011)$, tumor stage $(P=0.014)$, and preoperative carcinoembryonic antigen $(P<0.001)$. A negative correlation was found between PHGDH expression and the 5-year survival rate of patients with GC. Furthermore, multivariate analysis indicated that PHGDH was an independent prognostic factor for outcome in GC.

Conclusion: $\mathrm{PHGDH}$ is important in predicting patient outcomes and is a potential target for the development of therapeutic approaches to GC.

Keywords: metabolism, gastric cancer, prognosis, serine biosynthesis

\section{Introduction}

Gastric cancer (GC) is the fourth most prevalent human cancer worldwide, with an estimated 952,000 new cases diagnosed annually and almost as many deaths. ${ }^{1}$ Approximately $70 \%$ of GC cases are seen in developing countries. ${ }^{2}$ In the People's Republic of China, GC is the third leading cause of death among cancers, with an age-standardized incidence of 22.7/100,000. ${ }^{3}$ Unfortunately, the 5-year survival rate worldwide after surgical resection remains low. ${ }^{4}$ High rates of metastasis and recurrence are major obstacles in improving long-term survival after a curative resection. ${ }^{5}$ New molecular prognostic markers and therapeutic targets are needed to improve the clinical outcome for patients with this disease.

Over the past 10-20 years, there has been an increasing amount of evidence that the majority of oncogenes and tumor suppressors play vital roles in the regulation of metabolic processes. ${ }^{6}$ Tumor growth, for example, appears to be maintained through the "Warburg effect", which allows tumor cells to rely on aerobic glycolysis to maintain cell growth and proliferation. ${ }^{7}$ Furthermore, metabolic reprogramming of tumorigenic cells through modifications in the genome of metabolic enzymes contributes to their
Department of General Surgery,

Affiliated Hospital of Nantong University,

20 Xisi Road, Nantong, Jiangsu 22600I,

People's Republic of China

Tel/fax +86 $51381 \mid 6$ II $\mid$

Email wuhan_m@hotmail.com 
biomass accumulation and proliferative growth, and there is experimental evidence that tumor cell growth can be suppressed by blocking the activity of its constituent metabolic enzymes. ${ }^{8}$ For any of these enzymes to be considered as a target for developing anticancer therapies, there must be evidence of a profound difference in the requirements for the activity of that enzyme in cancerous cells vs normal cells. ${ }^{9}$ Recently, researchers have focused on phosphoglycerate dehydrogenase (PHGDH), a key enzyme in the de novo biosynthesis of serine. Specifically, PHGDH catalyzes a rate-limiting step in the conversion of 3-phosphoglycerate to serine. ${ }^{10}$ In so doing, it diverts flux away from glycolysis by oxidizing 3-phosphoglycerate to release serine and glycine, thus allowing the rapid production of energy and metabolites required for the high rate of anabolism that drives a dramatically increased proliferation of cancer cells. ${ }^{11}$

Until now, a high level of PHGDH expression has been detected in several types of human tumors; this appears to contribute to pathogenesis and to a poor prognosis for humans with cancer. ${ }^{12,13}$ In glioma cells, for example, PHGDH interacts with and stabilizes forkhead box M1, thereby promoting the proliferation, invasiveness, and tumorigenicity of the cell. ${ }^{14}$ Elevated rates of PHGDH expression have also been detected in cervical adenocarcinomas and have been found to correlate with advanced tumor stage, increased tumor size, and progression of the disease. ${ }^{15}$ Rotondo et $\mathrm{al}^{16}$ identified a similar trend in cervical neoplastic keratinocytes, and PHGDH appears to contribute to oncogenesis in breast cancer and melanoma cells. ${ }^{17}$ Conversely, a knockdown of endogenous PHGDH appears to promote apoptosis in human melanomas ${ }^{18}$ and inhibit HeLa cell proliferation while increasing sensitivity to cisplatin chemotherapy. ${ }^{19}$ Possemato et $\mathrm{al}^{20}$ found conclusive evidence that PHGDH levels are $\sim 70 \%$ higher in estrogen receptor-negative breast tumors than in estrogen receptor-positive breast tumors and account for an estimated $20 \%-25 \%$ of breast cancer cases and as many as $50 \%$ of breast cancer deaths within 5 years of diagnosis. ${ }^{21}$ A significant correlation between a high rate of PHGDH expression and a shorter time to relapse and worse overall survival (OS) was found in patients with breast cancer that had metastasized to bone. ${ }^{22}$ Combining key residues of protein kinase $\mathrm{C}(\mathrm{PKC}) \zeta$ to inhibit its enzymatic activity, phosphorylates PHGDH resulted in enhanced intestinal tumorigenesis and related with shorter OS in patients with low levels of $\mathrm{PKC} \zeta .{ }^{23}$ However, an analysis of data from patients with lung cancer did not demonstrate a relationship between PHGDH and prognosis, ${ }^{24}$ and a significantly increased level of PHGDH in tumoral vs peritumoral tissue failed to demonstrate any prognostic value in patients with colon cancer. ${ }^{25}$ Until now, the expression of PHGDH and its prognostic role in GC have not been explored. In this study, we sought to determine the prognostic value of various rates of PHGDH expression in benign tissue vs malignant tissue in patients with gastric disease.

\section{Methods}

\section{Human tissue specimens and patient clinical information}

We selected 617 formalin-fixed, paraffin-embedded tissue samples from patients with $\mathrm{GC}(\mathrm{n}=482)$, intestinal metaplasia $(n=38)$, low-grade intraepithelial neoplasia $(n=31)$, or highgrade intraepithelial neoplasia $(n=66)$. We also studied 20 samples of GC-involved tissue and paired tissue samples with normal margins. These tissue blocks were all obtained from the Department of Pathology, Affiliated Hospital of Nantong University, Jiangsu, People's Republic of China, between 2003 and 2010. The clinical characteristics of the patients with cancer were extracted from their medical records and included the age and sex of the patient, as well as the histological type, differentiation grade, and stage of the tumor, along with the preoperative serum carcinoembryonic antigen (CEA) and carbohydrate antigen-19-9 (CA19-9) levels. None of the patients with cancer had received any type of treatment before surgery. OS was defined as the interval between the initial biopsy-confirmed diagnosis and death or between the initial biopsy-confirmed diagnosis and the last follow-up for surviving patients. The study protocol was approved by the Human Research Ethics Committee of the Affiliated Hospital of Nantong University.

\section{Tissue microarray construction and immunohistochemistry analysis}

Tissue microarrays (TMAs) were constructed as previously described. ${ }^{3}$ In brief, TMAs were generated using the manual Tissue Microarrayer System Quick Ray (UT06; UNITMA, Seoul, South Korea). Immunohistochemical analysis was carried out using a mouse monoclonal antihuman PHGDH antibody $(5 \mu \mathrm{g} / \mathrm{mL}$ ) (Abcam, Cambridge, MA, USA). Reactions were detected using an EnVision $+{ }^{\mathrm{TM}}$ peroxidase kit (Dako Denmark A/S, Glostrup, Denmark). Samples were incubated with 3,3'-diaminobenzidine plus (Dako Denmark $\mathrm{A} / \mathrm{S}$ ) then counterstained with hematoxylin, dehydrated with graded alcohol, cleared in xylene, and coverslipped in a permanent mounting media.

Written informed consent was obtained from all study participants. All cases were reviewed and scored without knowledge of the clinical characteristics of the patients. PHGDH expression was scored using the semiquantitative 
$\mathrm{H}$-score method, taking into account both the staining intensity and the percentage of cells staining at that intensity. ${ }^{26}$ The staining intensity was scored as 0 (no stain), 1+ (weak stain), $2+$ (moderate stain), or $3+$ (intense stain). The percentage of cells staining at each intensity was determined and multiplied by the intensity score to produce an intensity percentage score. The final staining scores were calculated by adding the four intensity percentage scores. The staining score had a minimum value of 0 (no stain) and a maximum value of $300(100 \%$ of cells with a $3+$ staining intensity).

\section{Quantitative real-time polymerase chain reaction findings}

Fresh frozen tumor tissue samples $(n=20)$ and matched adjacent samples with normal margins were collected at the Department of Pathology, Affiliated Hospital of Nantong University, for quantitative real-time polymerase chain reaction analysis. Total tissue RNA was extracted using the RNeasy Mini Kit (Qiagen NV, Venlo, the Netherlands). Real-time polymerase chain reaction analysis was carried out according to the manufacturer's instructions (Quant SYBR Green PCR Kit; TIANGEN BIOTECH, Beijing, People's Republic of China). PHGDH (NM_006623) primer sequences were as follows: forward 5'-CACATTCTTGGGCTGAAC-3' and reverse 5'-TTATTAGACGGTTATTGCTGTA-3'. GAPDH was used as an internal control. For relative quantification, $2^{-\Delta \Delta \mathrm{Ct}}$ was calculated and used as an indicator of the level of enzyme expression.

\section{Statistical analysis}

The statistical analysis was carried out using the SPSS 22.0 statistical software package (IBM Corporation, Armonk, NY, USA). Cutoff values for high or low PHGDH expression were measured using the X-Tile software (Rimm Lab, Yale University School of Medicine, New Haven, USA; http://www.tissuearray.org/rimmlab). ${ }^{27,28}$ The relationship between PHGDH expression and clinicopathologic variables was estimated using the $\chi^{2}$ test, Fisher's exact test, or $t$-test, as appropriate. Survival curves were analyzed using the Kaplan-Meier method and compared by log-rank test. The Cox proportional hazard model was used for univariate and multivariate analyses, in which all of the clinicopathologic features served as covariates. Statistical significance was established at $P<0.05$ (two-tailed).

\section{Results \\ PHGDH expression in GC tissues}

We explored PHGDH expression by performing IHC analysis on TMA comprising 482 GC and 64 matched tumor adjacent tissues from patients with GC. We also examined PHGDH expression in 20 chronic gastritis, 18 intestinal metaplasia, and 31 low-grade and 66 high-grade intraepithelial neoplasias. IHC analysis of TMA sections showed that PHGDH was expressed mainly in the tumor epithelial cells, and staining showed that it occurred primarily in the cytoplasm (Figure 1). The X-Tile-based TMA data analysis indicated a significant cutoff point for OS in GC. For PHGDH, the cutoff selected was 120, ie, a score of 0-120 represented a low rate of expression (PHGDH-low) and a score of 121-300 represented a high rate of expression (PHGDH-high). Overall, only a small proportion of chronic gastritis $(20 \%, 4 / 20)$ and matched tumor adjacent tissues $(20.31 \%, 13 / 64)$ displayed high PHGDH expression, whereas high expression of this protein was detected in $50.0 \%(9 / 18)$ of intestinal metaplasia, $45.16 \%(14 / 31)$ of low-grade intraepithelial neoplasia, 56.06\% (37/66) of high-grade intraepithelial neoplasia, and 45.64\% (220/482) of GCs $(P<0.001)$ (Table 1).

To investigate the difference in the PHGDH gene expression levels between cancerous and normal tissues, total RNA was extracted from fresh GC cancer tissues and matched tumor adjacent tissue samples $(n=20)$. PHGDH expression was significantly higher in the cancerous tissue samples than in their paired peritumoral counterparts $(P=0.007)$ (Figure 2A).

\section{Relationship between PHGDH expression and clinicopathologic characteristics in GC}

To determine whether PHGDH is important in determining clinical outcomes for patients with GC, we examined the relationship between rates of PHGDH expression and clinical parameters in these patients. PHGDH-high expression correlated significantly with the patient's age ( $P=0.009)$; tumor histology $(P=0.011)$, stage $(P=0.014)$, and classification $(P=0.043)$; preoperative CEA $(P=0.016)$ and CA19-9 $(P=0.014)$ levels; and evidence of lymph node metastasis $(P=0.024)$ and tumor metastasis $(P=0.005)$ (Table 2).

\section{Prognostic value of PHGDH expression in GC}

On examining the relationship between rates of PHGDH expression and prognostic data, we found that patients with GC with PHGDH-high had a significantly worse prognosis than those with PHGDH-low. The 1-, 3-, and 5-year OS for PHGDH-high patients with GC was worse than that for their PHGDH-low counterparts $(P<0.001)$ (Figure 2B). 


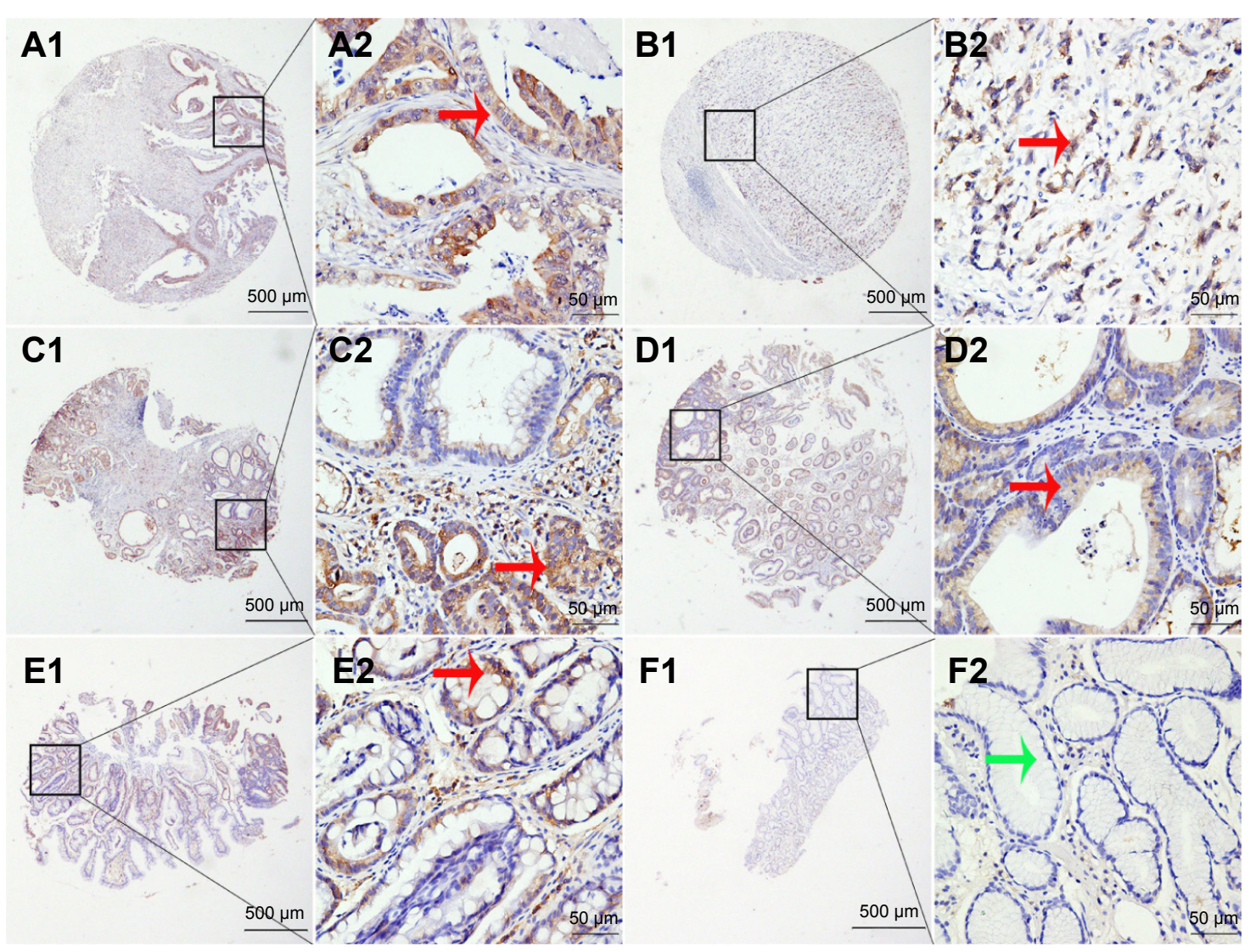

Figure I PHGDH protein expression in benign and malignant gastric tissue samples in TMA sections.

Notes: (Al and A2) Well-differentiated gastric cancer tissue with moderate PHGDH expression; (B I and B2) poorly differentiated gastric cancer tissue with strong PHGDH expression; (CI and C2) High-grade intraepithelial neoplasia with moderate PHGDH expression; (DI and D2) Low-grade intraepithelial neoplasia with weak PHGDH expression; (EI and E2) Intestinal metaplasia with weak PHGDH expression; (FI and F2) Normal surgical margin of gastric cancer, negative. Original magnification $\times 40$ (bar =500 $\mu \mathrm{m}$ ) in (AI), (BI), (CI), (DI), (EI), and (FI) and $\times 400$ (bar =50 $\mu \mathrm{m})$ in (A2), (B2), (C2), (D2), (E2), and (F2). Red arrows indicate positive PHGDH staining while green arrows indicate negative PHGDH staining.

Abbreviations: PHGDH, phosphoglycerate dehydrogenase; TMA, tissue microarray.

We also evaluated prognostic factors for patients with GC using both univariate and multivariate analyses. The univariate analysis revealed that PHGDH-high correlated significantly with a poor OS (hazard ratio [HR]: 2.833; 95\% CI: $2.209-3.633 ; P<0.001)$ and with previously reported prognostic markers, including differentiation (HR: $1.537 ; 95 \%$ CI: $1.193-1.979 ; P<0.001$ ), tumor stage (HR: 1.588 ; 95\% CI: $1.47-1.715 ; P<0.001$ ), preoperative
CEA (HR: 2.227; 95\% CI: 1.571-3.159; $P<0.001$ ), and preoperative CA19-9 (HR: 2.498; 95\% CI: 1.705-3.66; $P<0.001$ ) (Table 3). The multivariate analysis also revealed that PHGDH-high correlated significantly with a worse OS (HR: 2.605 ; 95\% CI: $1.802-3.767 ; P<0.001$ ) as well as with advanced tumor stage (HR: $1.62 ; 95 \%$ CI: $1.433-1.83$; $P<0.001)$ and preoperative CEA (HR: $1.787 ; 95 \% \mathrm{CI}$ : 1.192-2.68; $P=0.005$ ) (Table 3). Preoperative CEA levels

Table I PHGDH expression in gastric benign and malignant tissues

\begin{tabular}{|c|c|c|c|c|c|}
\hline Characteristic & $\mathbf{n}$ & PHGDH- (\%) & PHGDH+ (\%) & Pearson $\chi^{2}$ & $P$-value \\
\hline Stomach & 681 & & & 25.613 & $<0.00 I^{*}$ \\
\hline Chronic gastritis & 20 & $16(80.00)$ & $4(20.00)$ & & \\
\hline Intestinal metaplasia & 18 & $9(50.00)$ & $9(50.00)$ & & \\
\hline Low-grade intraepithelial neoplasia & 31 & $17(54.84)$ & $14(45.16)$ & & \\
\hline High-grade intraepithelial neoplasia & 66 & $29(43.94)$ & $37(56.06)$ & & \\
\hline Cancer & 482 & $262(54.36)$ & $220(45.64)$ & & \\
\hline Surgical margin & 64 & 51 (79.69) & $13(20.3 \mathrm{I})$ & & \\
\hline
\end{tabular}

Notes: PHGDH- indicates low PHGDH expression, and PHGDH+ indicates high PHGDH expression. $* P<0.05$. The data were analyzed by ANOVA with SPSS 20.0 statistical software.

Abbreviation: $\mathrm{PHGDH}$, phosphoglycerate dehydrogenase. 

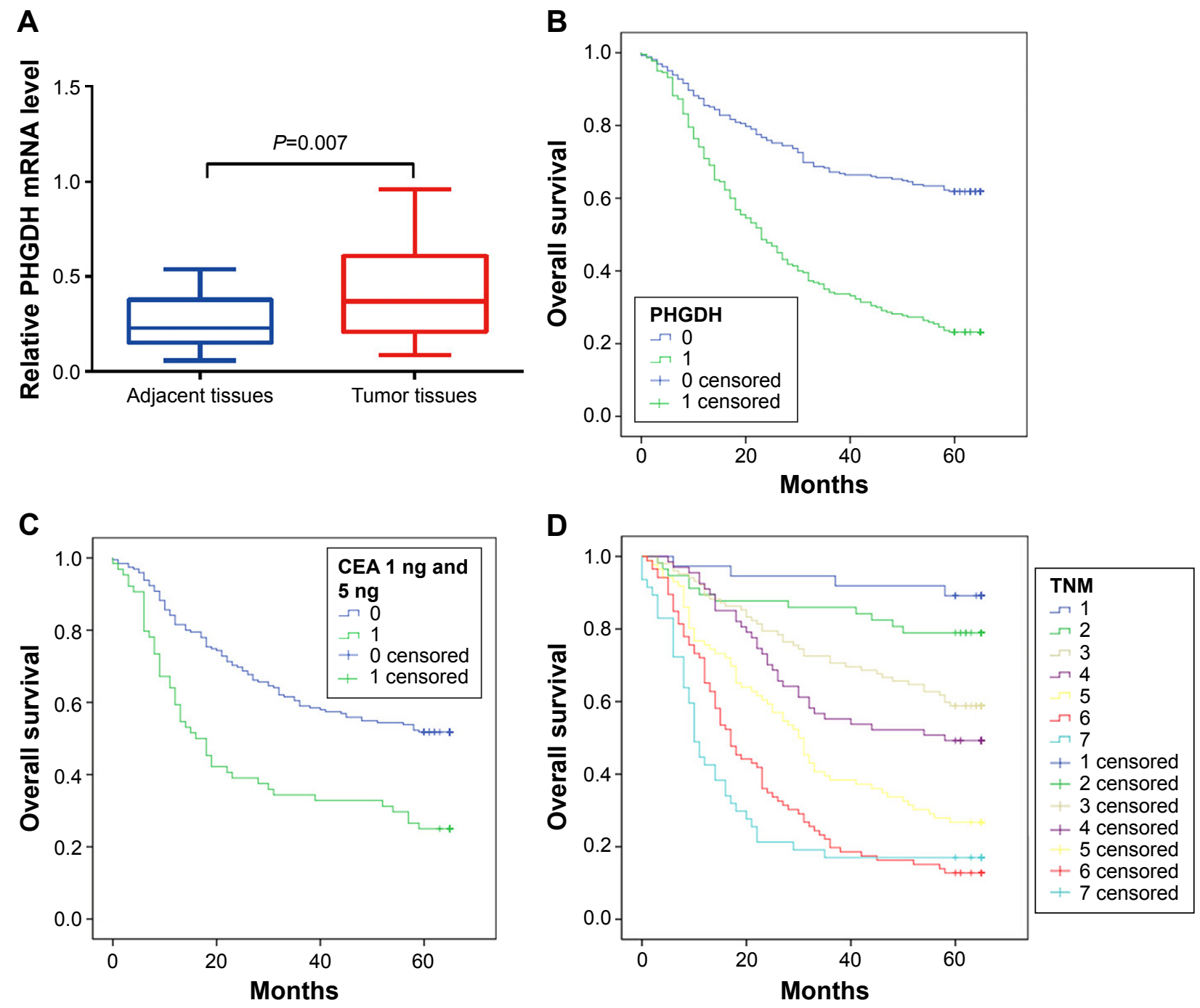

Figure 2 Kaplan-Meier survival curves for gastric cancer, with comparisons evaluated using the log-rank test.

Notes: (A) PHGDH mRNA levels in GC specimens determined by real-time PCR. PHGDH mRNA was significantly higher in GC tissues than in their paired peritumoral counterparts $(P=0.007)$. (B) Overall survival was significantly shorter in patients with PHGDH-high expression (green line: I) vs PHGDH-low expression (blue line: 0). (C) Overall survival was significantly shorter in patients with preoperative CEA-high levels (green line: I) vs CEA-low levels (blue line: 0). (D) Advanced TNM stage significantly worsened overall survival compared with early TNM stage: TNM IIlc and IV (light blue line: 7), TNM IIlb (red line: 6), TNM Illa (yellow line: 5), TNM IIb (purple line: 4), TNM lla (light yellow line: 3), TNM lb (green line: 2), and TNM la +0 (blue line: I).

Abbreviations: CEA, carcinoembryonic antigen; GC, gastric cancer; PCR, polymerase chain reaction; PHGDH, phosphoglycerate dehydrogenase; mRNA, messenger RNA; TNM, tumor node metastasis.

$(>5 \mathrm{ng} / \mathrm{mL} ; P<0.001)$ and tumor node metastasis $(\mathrm{TNM})$ stage $(P<0.001)$ also individually influenced survival, with low preoperative CEA levels and early TNM stage predicting a favorable prognosis (Figure 2C and D).

\section{Discussion}

Here, we report the findings of the first large-scale study to use high-throughput TMA analyses to examine the prognostic value of PHGDH expression in tumors in a random population of patients with gastric disease. In this study, we found that PHGDH, the key enzyme involved in de novo serine biosynthesis, is significantly elevated in gastric tumor specimens compared with normal gastric epithelial specimens. An increased rate of expression of PHGDH in GC correlated with a more aggressive tumor behavior, a more cancerous histological type, a more advanced stage of disease, and a greater risk of lymph node metastasis.

We also found a strong correlation between PHGDH-high expression and a reduced OS in patients with GC. A multivariate analysis provided further support for the value of PHGDH as an independent prognostic factor, along with such wellestablished factors as tumor stage. However, such evidence has not been consistent. For example, patients with a high rate of PHGDH expression are prone to a worse prognosis with an advanced tumor stage alone; this is in concordance with most previous studies of other cancers. Additionally, PHGDH reportedly has no prognostic value in patients with colon cancer, ${ }^{25}$ and its prognostic value in breast cancer has been 
Table 2 Association of high expression of PHGDH with clinicopathological characteristics in patients with gastric cancer

\begin{tabular}{|c|c|c|c|c|c|}
\hline Characteristic & $\mathbf{n}$ & $\begin{array}{l}\text { PHGDH-, } \\
\text { n (\%) }\end{array}$ & $\begin{array}{l}\text { PHGDH+, } \\
\text { n (\%) }\end{array}$ & $\begin{array}{l}\text { Pearson } \\
\chi^{2}\end{array}$ & $P$-value \\
\hline Total & 482 & $262(54.36)$ & $220(45.64)$ & & \\
\hline Sex & & & & 1.836 & 0.175 \\
\hline Male & 356 & $187(52.53)$ & $169(47.47)$ & & \\
\hline Female & 126 & 75 (59.52) & $51(40.48)$ & & \\
\hline Age (years) & & & & 6.768 & $0.009 *$ \\
\hline$<60$ & 170 & $106(62.35)$ & $64(37.65)$ & & \\
\hline$\geq 60$ & 312 & $156(50.00)$ & $156(50.00)$ & & \\
\hline Histological type & & & & 11.239 & $0.011 *$ \\
\hline Tubular & 390 & $207(53.08)$ & $183(46.92)$ & & \\
\hline $\begin{array}{l}\text { Mixed (tubular } \\
\text { and mucinous) }\end{array}$ & 17 & $12(70.95)$ & $5(29.4 I)$ & & \\
\hline Mucinous & 27 & $14(5 \mid .85)$ & $13(48.15)$ & & \\
\hline Signet ring cell & 17 & $15(88.24)$ & $2(11.76)$ & & \\
\hline Others ${ }^{\mathrm{a}}$ & 31 & $17(54.84)$ & $14(45.16)$ & & \\
\hline Differentiation & & & & 2.448 & 0.294 \\
\hline Well & 13 & $7(53.85)$ & $6(46.15)$ & & \\
\hline Middle & 134 & $67(50.50)$ & $67(50.50)$ & & \\
\hline Poor & 305 & $177(58.03)$ & $128(41.97)$ & & \\
\hline Others $^{\mathrm{b}}$ & 30 & $19(63.33)$ & II (36.67) & & \\
\hline TNM stage & & & & 15.907 & $0.014 *$ \\
\hline $0+$ la & 37 & 29 (8I.48) & $8(18.52)$ & & \\
\hline $\mathrm{lb}$ & 57 & $35(61.40)$ & $22(38.60)$ & & \\
\hline Ila & 102 & $58(56.86)$ & $44(43.14)$ & & \\
\hline Ilb & 67 & 37 (55.22) & $30(44.78)$ & & \\
\hline IIla & 86 & $43(50.00)$ & $43(50.00)$ & & \\
\hline Illb & 86 & $4 \mathrm{I}(47.67)$ & $45(52.33)$ & & \\
\hline IIIc + IV & 47 & $19(40.43)$ & $28(59.57)$ & & \\
\hline $\mathrm{T}$ & & & & 8.138 & $0.043^{*}$ \\
\hline Tis & 10 & $7(70.00)$ & $3(30.00)$ & & \\
\hline TI & 41 & $30(73.17)$ & II (26.83) & & \\
\hline $\mathrm{T} 2$ & 100 & $54(54.00)$ & $46(46.00)$ & & \\
\hline $\mathrm{T} 3+\mathrm{T} 4$ & 331 & $|7|(5 \mid .66)$ & $160(48.34)$ & & \\
\hline $\mathrm{N}$ & & & & 9.455 & $0.024 *$ \\
\hline No & 179 & $110(61.45)$ & $69(38.55)$ & & \\
\hline $\mathrm{NI}$ & 91 & $53(58.24)$ & $38(41.76)$ & & \\
\hline N2 & 101 & $49(48.5 I)$ & $52(51.49)$ & & \\
\hline N3 & 111 & $50(45.05)$ & 61 (54.95) & & \\
\hline$M$ & & & & 7.765 & $0.005^{*}$ \\
\hline MO & 445 & $250(56.18)$ & 195 (43.82) & & \\
\hline MI & 37 & $12(32.43)$ & $25(67.57)$ & & \\
\hline Preoperative & & & & 13.068 & $<0.00 I^{*}$ \\
\hline \multicolumn{6}{|l|}{ CEA $(\mathrm{ng} / \mathrm{mL})$} \\
\hline$\leq 5$ & 195 & $129(66.15)$ & $66(33.85)$ & & \\
\hline$>5$ & 64 & $26(40.63)$ & $38(59.38)$ & & \\
\hline Unknown & 223 & $126(56.50)$ & $97(43.50)$ & & \\
\hline Preoperative & & & & 6.080 & $0.014 *$ \\
\hline \multicolumn{6}{|l|}{ CAI9-9 (U/mL) } \\
\hline$\leq 37$ & 207 & $133(64.25)$ & $74(35.75)$ & & \\
\hline$>37$ & 45 & $20(44.44)$ & $25(55.56)$ & & \\
\hline Unknown & 230 & $145(63.04)$ & $85(36.96)$ & & \\
\hline
\end{tabular}

Notes: apapillary adenocarcinoma, three cases; adenosquamous carcinoma, three cases; squamous cell carcinoma, three cases; undifferentiated carcinoma, one case; neuroendocrine carcinoma, one case; small cell osteosarcoma, seven cases; carcinoid, one case; focal cancer, twelve cases. ${ }^{\mathrm{T} T u b u l a r}$ and papillary adenocarcinoma. ${ }^{* P}<0.05$. The data were analyzed by ANOVA with SPSS 20.0 statistical software.

Abbreviations: CAI9-9, carbohydrate antigen-19-9; CEA, carcinoembryonic antigen; PHGDH, phosphoglycerate dehydrogenase; TNM, tumor node metastasis. contradictory. ${ }^{24,29}$ However, its expression in the epithelium of the small intestine, ${ }^{23}$ in cervical adenocarcinomas, ${ }^{19}$ and in GC tissue (our data) has been associated with a poor prognosis. Furthermore, a recent study showed PHGDH expression in the stroma with an increased tumor grade and a reduced disease-free survival in phyllodes tumor. ${ }^{30}$ The contradictory nature of findings regarding the prognostic value of PHGDH is exemplified in the studies of lobular vs invasive ductal carcinoma, with high levels of PHGDH found in invasive lobular carcinoma compared with invasive ductal carcinoma, but higher rates of expression of metabolismrelated proteins found in invasive ductal carcinoma tissue compared with invasive stromal lobular carcinoma tissue. ${ }^{31}$ These observations suggest different expression patterns and roles for PHGDH in different types of cancer.

Before PHGDH can be investigated for its potential as a therapeutic target, a significant difference must be found in the requirement for PHGDH activity in cancerous vs normal proliferating cells. ${ }^{6,32} \mathrm{We}$ found that PHGDH expression is significantly higher in $\mathrm{GC}$ tissue compared with benign or normal tissue. This strongly implicates PHGDH as an attractive target for anticancer drug development for patients with the subset of tumors that amplify and overexpress its gene. The broader network of metabolic mechanisms involved in cancer must be studied to determine the potential for PHGDH as a metabolic oncogene. ${ }^{29,30}$ Serine biosynthesis was found to correlate with $\mathrm{p} 73$ expression in human lung adenocarcinomas. ${ }^{9}$ Regulation of PHGDH by EBV-miR-BART1 in nasopharyngeal carcinomas might involve the concomitant overexpression of p130Cas and ERBB2 activation. ${ }^{33}$ In mice lacking the repressive effect of $\mathrm{PKC} \zeta$, the rate of both tumorigenesis and PHGDH expression increases. ${ }^{23}$ In prostate cancer, PHGDH has been found to regulate c-Myc phosphorylation, ${ }^{34}$ and in human intestinal tumor, it correlates with caspase- $3 .{ }^{23}$ Tagging PHGDH limits its ability to convert 3-phosphoglycerate (3PG) to phosphohydroxypyruvate (PHP). Tagging may involve the addition of an N-terminal epitope to PHGDH to disrupt its ability to sustain cancer cell proliferation. ${ }^{35}$ The findings of many recent studies suggest that PHGDH inhibition is a viable mechanism for developing anticancer agents for two reasons. ${ }^{36}$ First, a PHGDH inhibitor that cannot cross the blood-brain barrier would not interfere with serine homeostasis in the central nervous system; this would prevent the neurological effects that have been reported for known PHGDH mutations in humans. Second, serine deficiency disorders can be treated using exogenous serine supplements, and the need for PHGDH in tumors might not be associated with a serine flux. ${ }^{8}$ Encouragingly, a nonmetabolic role for PHGDH in glioma 
Table 3 Univariate and multivariate analysis of prognostic factors for overall survival in patients with gastric cancer

\begin{tabular}{|c|c|c|c|c|c|c|c|c|}
\hline \multirow[t]{2}{*}{ Variable } & \multicolumn{4}{|c|}{ Univariate analysis } & \multicolumn{4}{|c|}{ Multivariate analysis } \\
\hline & HR & $P>|z|$ & $95 \% \mathrm{Cl}$ & & HR & $P>|z|$ & $95 \% \mathrm{C}$ & \\
\hline \multicolumn{9}{|l|}{ PHGDH expression } \\
\hline High vs low & 2.833 & $<0.00 I^{*}$ & 2.209 & 3.633 & 2.605 & $<0.00 I^{*}$ & 1.802 & 3.767 \\
\hline \multicolumn{9}{|l|}{ Age (years) } \\
\hline$\leq 60$ vs $>60$ & 1.254 & 0.084 & 0.970 & 1.621 & & & & \\
\hline \multicolumn{9}{|l|}{ Sex } \\
\hline Male vs female & 0.944 & 0.676 & 0.721 & 1.236 & & & & \\
\hline \multicolumn{9}{|l|}{ Histological type } \\
\hline $\begin{array}{l}\text { Tubular vs mixed (tubular and mucinous) vs } \\
\text { mucinous vs signet ring cell carcinoma vs others }{ }^{\mathrm{a}}\end{array}$ & 0.950 & 0.357 & 0.853 & 1.059 & & & & \\
\hline \multicolumn{9}{|l|}{ Differentiation } \\
\hline Well vs middle vs poor & 1.537 & $0.001 *$ & 1.193 & 1.979 & 1.285 & 0.197 & 0.878 & 1.881 \\
\hline \multicolumn{9}{|l|}{ TNM stage } \\
\hline $0+$ la vs Ib vs Ila vs Ilb vs IIla vs IIIb vs IIlc + IV & 1.588 & $<0.00 I^{*}$ & 1.470 & 1.715 & 1.620 & $<0.00 I^{*}$ & 1.433 & 1.830 \\
\hline \multicolumn{9}{|l|}{$\mathrm{T}$} \\
\hline Tis vs $\mathrm{TI}$ vs $\mathrm{T} 2$ vs $\mathrm{T} 3+\mathrm{T} 4$ & 2.206 & $<0.00 I^{*}$ & 1.621 & 2.533 & & & & \\
\hline \multicolumn{9}{|l|}{$\mathrm{N}$} \\
\hline No vs N2 vs N3 & 1.691 & $<0.00 I^{*}$ & 1.530 & 1.869 & & & & \\
\hline \multicolumn{9}{|l|}{ M } \\
\hline M0 vs MI & 3.191 & $<0.00 I^{*}$ & 2.188 & 4.654 & & & & \\
\hline \multicolumn{9}{|l|}{ Preoperative CEA (ng/mL) } \\
\hline$\leq 5$ vs $\geq 5$ & 2.227 & $<0.001 *$ & $1.57 \mid$ & 3.159 & 1.787 & $0.005^{*}$ & 1.192 & 2.680 \\
\hline \multicolumn{9}{|l|}{ Preoperative CAI9-9 (U/mL) } \\
\hline$\leq 37$ vs $>37$ & 2.498 & $<0.00 \mathrm{I} *$ & $\mathrm{I} .705$ & 3.660 & 1.514 & 0.062 & 0.980 & 2.339 \\
\hline
\end{tabular}

Notes: aPapillary adenocarcinoma, three cases; adenosquamous carcinoma, three cases; squamous cell carcinoma, three cases; undifferentiated carcinoma, one case; neuroendocrine carcinoma, one case; small cell osteosarcoma, seven cases; carcinoid, one case; focal cancer, twelve cases. $* P<0.05$.

Abbreviations: CA19-9, carbohydrate antigen-19-9; CEA, carcinoembryonic antigen; HR, hazard ratio; PHGDH, phosphoglycerate dehydrogenase.

tumorigenesis has been reported ${ }^{14}$ and may provide a novel mechanism for developing antitumor therapies.

There are several limitations in our study. First, it is a retrospective observational study; thus, the findings might not be applicable to the general population. Larger prospective studies are needed to confirm our results. Second, the immunohistochemistry data are semiquantitative; additional methods are needed to evaluate and confirm the rates of PHGDH expression in tumor cells. Third, in vitro studies are needed to investigate the mechanism(s) underlying PHGDH activity in tumorigenesis.

\section{Conclusion}

We have shown that a higher rate of PHGDH expression is an independent marker of prognosis in GC. Because of its role in tumor-associated serine biosynthesis, future research is warranted to investigate the potential mechanisms by which PHGDH promotes cancer, with the aim of using it as a target for developing new therapeutic approaches to cancer.

\section{Acknowledgments}

This study was funded by National Natural Science Foundation of China (grant 81572390) and Project of Nantong Science and Technology Bureau, Jiangsu Province, People's Republic of China (number MS22015116).

\section{Disclosure}

The authors report no conflicts of interest in this work.

\section{References}

1. Alexandrov LB, Nik-Zainal S, Siu HC, Leung SY, Stratton MR. A mutational signature in gastric cancer suggests therapeutic strategies. Nat Commun. 2015;6:8683.

2. Ito S, Oki E, Nakashima Y, et al. Clinical significance of adjuvant surgery following chemotherapy for patients with initially unresectable stage IV gastric cancer. Anticancer Res. 2015;35(1):401-406.

3. Sun R, Wang X, Zhu H, et al. Prognostic value of LAMP3 and TP53 overexpression in benign and malignant gastrointestinal tissues. Oncotarget. 2014;5(23):12398-12409.

4. Wang Z, Liu H, Shen Z, et al. The prognostic value of CXC-chemokine receptor 2 (CXCR2) in gastric cancer patients. BMC Cancer. 2015; 15:766.

5. Fukuda Y, Yamamoto K, Hirao M, et al. Sarcopenia is associated with severe postoperative complications in elderly gastric cancer patients undergoing gastrectomy. Gastric Cancer. 2016;19(3):986-993.

6. Fan J, Teng X, Liu L, et al. Human phosphoglycerate dehydrogenase produces the oncometabolite D-2-hydroxyglutarate. ACS Chem Biol. 2015; 10(2):510-516.

7. Sciacovelli M, Gaude E, Hilvo M, Frezza C. The metabolic alterations of cancer cells. Methods Enzymol. 2014;542:1-23.

8. Luo J. Cancer's sweet tooth for serine. Breast Cancer Res. 2011;13(6): 317. 
9. Amelio I, Markert EK, Rufini A, et al. p73 regulates serine biosynthesis in cancer. Oncogene. 2014;33(42):5039-5046.

10. Zogg CK. Phosphoglycerate dehydrogenase: potential therapeutic target and putative metabolic oncogene. J Oncol. 2014;2014:524101.

11. Mullarky E, Mattaini KR, Vander Heiden MG, Cantley LC, Locasale JW. PHGDH amplification and altered glucose metabolism in human melanoma. Pigment Cell Melanoma Res. 2011;24(6):1112-1115.

12. Gromova I, Gromov P, Honma N, et al. High level PHGDH expression in breast is predominantly associated with keratin 5-positive cell lineage independently of malignancy. Mol Oncol. 2015;9(8):1636-1654.

13. Chen J, Chung F, Yang G, et al. Phosphoglycerate dehydrogenase is dispensable for breast tumor maintenance and growth. Oncotarget. 2013; 4(12):2502-2511.

14. Liu J, Guo S, Li Q, et al. Phosphoglycerate dehydrogenase induces glioma cells proliferation and invasion by stabilizing forkhead box M1. J Neurooncol. 2013;111(3):245-255.

15. Jing Z, Heng W, Aiping D, Yafei Q, Shulan Z. Expression and clinical significance of phosphoglycerate dehydrogenase and squamous cell carcinoma antigen in cervical cancer. Int J Gynecol Cancer. 2013;23(8): 1465-1469.

16. Rotondo JC, Bosi S, Bassi C, et al. Gene expression changes in progression of cervical neoplasia revealed by microarray analysis of cervical neoplastic keratinocytes. J Cell Physiol. 2015;230(4):806-812.

17. Locasale JW, Grassian AR, Melman T, et al. Phosphoglycerate dehydrogenase diverts glycolytic flux and contributes to oncogenesis. Nat Genet. 2011;43(9):869-874.

18. Ou Y, Wang SJ, Jiang L, Zheng B, Gu W. p53 Protein-mediated regulation of phosphoglycerate dehydrogenase (PHGDH) is crucial for the apoptotic response upon serine starvation. J Biol Chem. 2015;290(1): $457-466$.

19. Jing Z, Heng W, Xia L, et al. Downregulation of phosphoglycerate dehydrogenase inhibits proliferation and enhances cisplatin sensitivity in cervical adenocarcinoma cells by regulating Bcl-2 and caspase-3. Cancer Biol Ther. 2015;16(4):541-548.

20. Possemato R, Marks KM, Shaul YD, et al. Functional genomics reveal that the serine synthesis pathway is essential in breast cancer. Nature. 2011;476(7360):346-350.

21. van de Vijver MJ, He YD, van't Veer LJ, et al. A gene-expression signature as a predictor of survival in breast cancer. $N$ Engl J Med. 2002;347(25):1999-2009.

22. Pollari S, Kakonen SM, Edgren H, et al. Enhanced serine production by bone metastatic breast cancer cells stimulates osteoclastogenesis. Breast Cancer Res Treat. 2011;125(2):421-430.
23. Ma L, Tao Y, Duran A, et al. Control of nutrient stress-induced metabolic reprogramming by PKCzeta in tumorigenesis. Cell. 2013;152(3): 599-611.

24. Antonov A, Agostini M, Morello M, Minieri M, Melino G, Amelio I. Bioinformatics analysis of the serine and glycine pathway in cancer cells. Oncotarget. 2014;5(22):11004-11013.

25. Yoon S, Kim JG, Seo AN, et al. Clinical implication of serine metabolismassociated enzymes in colon cancer. Oncology. 2015;89(6):351-359.

26. Detre S, Saclani Jotti G, Dowsett M. A “quickscore" method for immunohistochemical semiquantitation: validation for oestrogen receptor in breast carcinomas. J Clin Pathol. 1995;48(9):876-878.

27. Huang J, Fan X, Wang X, et al. High ROR2 expression in tumor cells and stroma is correlated with poor prognosis in pancreatic ductal adenocarcinoma. Sci Rep. 2015;5:12991.

28. Lu C, Wang X, Zhu H, Feng J, Ni S, Huang J. Over-expression of ROR2 and Wnt5a cooperatively correlates with unfavorable prognosis in patients with non-small cell lung cancer. Oncotarget. 2015;6(28): 24912-24921.

29. Kim SK, Jung WH, Koo JS. Differential expression of enzymes associated with serine/glycine metabolism in different breast cancer subtypes. PLoS One. 2014;9(6):e101004.

30. Kwon JE, Kim DH, Jung WH, Koo JS. Expression of serine and glycine-related enzymes in phyllodes tumor. Neoplasma. 2014;61(5): 566-578.

31. Kim YH, Jung WH, Koo JS. Expression of metabolism-related proteins in invasive lobular carcinoma: comparison to invasive ductal carcinoma. Tumour Biol. 2014;35(10):10381-10393.

32. Hamanaka RB, Chandel NS. Targeting glucose metabolism for cancer therapy. J Exp Med. 2012;209(2):211-215.

33. Ye Y, Zhou Y, Zhang L, et al. EBV-miR-BART1 is involved in regulating metabolism-associated genes in nasopharyngeal carcinoma. Biochem Biophys Res Commun. 2013;436(1):19-24.

34. Kim JY, Valencia T, Abu-Baker S, et al. c-Myc phosphorylation by PKCzeta represses prostate tumorigenesis. Proc Natl Acad Sci US A. 2013;110(16):6418-6423.

35. Mattaini KR, Brignole EJ, Kini M, et al. An epitope tag alters phosphoglycerate dehydrogenase structure and impairs ability to support cell proliferation. Cancer Metab. 2015;3:5.

36. Erez A, DeBerardinis RJ. Metabolic dysregulation in monogenic disorders and cancer - finding method in madness. Nat Rev Cancer. 2015; 15(7):440-448.
OncoTargets and Therapy

\section{Publish your work in this journal}

OncoTargets and Therapy is an international, peer-reviewed, open access journal focusing on the pathological basis of all cancers, potential targets for therapy and treatment protocols employed to improve the management of cancer patients. The journal also focuses on the impact of management programs and new therapeutic agents and protocols on

\section{Dovepress}

patient perspectives such as quality of life, adherence and satisfaction. The manuscript management system is completely online and includes a very quick and fair peer-review system, which is all easy to use. Visit http://www.dovepress.com/testimonials.php to read real quotes from published authors. 